\title{
Reduction of rating scale data by means of signal detection theory
}

\author{
MANFRED VELDEN \\ Universitat Mainz, 6500 Mainz, West Germany \\ and \\ W. CRAWFORD CLARK \\ New York State Psychiatric Institute, New York, New York 10032
}

\begin{abstract}
A $d$ '-index computed from ratings of psychological stimuli does not necessarily represent the unbiased psychological distance between these stimuli. Such ratings may not be considered as discrimination tasks in the signal detection sense, because the rater has to discriminate between the psychological impact of the stimuli on some internal continuum rather than between the stimuli as such. The rater therefore can use stimulus-specific criteria for his decisions. As a result, the $\mathrm{d}^{\prime}$-index computed from the rating data will be biased.
\end{abstract}

In the psychophysics of detection and discrimination, the problem of response bias has been largely resolved by means of signal detection theory (SDT). This theory allows non-sensory influences (response bias) to be isolated from a subject's ability to detect or to discriminate between stimuli. The formal analogy between the SDT rating experiment (Green \& Swets, 1966 ) and the ratings that are commonly used in psychology (e.g., self-ratings) allow reduction of the data from such ratings on the basis of SDT. This procedure has been used by several researchers in order to control response bias which is often a critical problem with rating scale data (Chapman \& Feather, 1971; Clark, Kurlander, Bieber, \& Glassman, 1976; Dorfman \& Saslow, 1972; Grossberg \& Grant, 1978; Neufeld, 1975; Chapman \& Gentry, Note 1).

In order to illustrate the application of SDT to rating data, let us simplify the rating procedure such that there are only two rating categories left, for example "high" and "low" if stimuli are rated with respect to some psychological continuum, or "true" and "false" in case of questionnaire items.

Figure 1 shows the psychophysical model of the SDT discrimination experiment. Concerning the application of this model to common ratings, imagine two psychological objects or stimuli, $S_{1}$ and $S_{2}$ (e.g., pictures of homicide victims), that are to be rated with respect to some psychological variable (e.g., aversiveness). There is a mean value for each of these stimuli, $\mu_{1}$ and $\mu_{2}$, on the psychological continuum (observation axis, $x$, in the SDT nomenclature) and two distributions of possible values for each stimulus,

Requests for reprints should be sent to M. Velden, Psychologisches Institut, Universität Mainz, Saarstr. 21, 6500 Mainz, West Germany. $f\left(x \mid S_{1}\right)$ and $f\left(x \mid S_{2}\right)$. If the experienced aversiveness of a picture exceeds the value $x_{c}$ (criterion) for some rater, then he or she responds with "high," if it is less, he or she gives the rating "low." The parameter $\mathrm{d}$ ' represents the difference between the two means, $\mu_{2}-\mu_{1}$, and thus the sensory distance between the stimuli. In case of questionnaire ratings or self-ratings, we may think of two sets of items, for example, items that describe a tense state $\left(\mathrm{S}_{1}\right)$ and items that describe a relaxed state $\left(S_{2}\right)$. The internal continuum ( $x$ ) would now be "trueness" (with respect to the state of the rater). If the subjective trueness of an item exceeds $x_{c}$, then the rater responds with "true," otherwise with "false." For the example given, positive $d$ ' values will indicate a rather relaxed state of the rater, because the distribution for the relaxed items is located higher on the trueness continuum. Negative ones will indicate a rather tense state.

The question arises whether $d^{\prime}$, when computed from psychological ratings, may be interpreted in the SDT sense, that is to say as reflecting the mean sensory distance between two stimuli or groups of

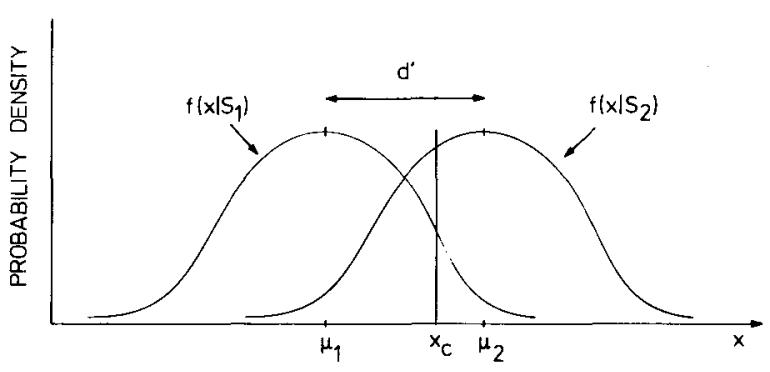

Figure 1. Psychophysical model of SDT discrimination experiment. 
stimuli free of response bias. In order to answer this question, it is important to note a critical difference between the SDT discrimination experiment and the rating of psychological objects, that is, objects that cannot be readily calibrated on a physical scale. In case of the SDT experiment, the subject's task is to discriminate between physical stimuli on the basis of values on the observation axis evoked by different physical quantities. These values are the sole sensory basis of the subject's decisions. However, in the case of psychological stimuli, it is not a physical quantity but some psychologically relevant content or aspect that is the entity to be rated. The subject's task is not to discriminate between the stimuli as such, but only to discriminate between their psychological impact as represented on some internal psychological continuum. Thus, even though the distributions of values on this continuum, resulting from the application of the stimuli, may overlap, the subject can perfectly discriminate between the stimuli as such. For the experimenter, there is no way to isolate the relevant psychological aspect of the stimuli and present it alone to the subjects. As a consequence, the experimenter cannot be sure that a subject bases his or her rating decisions solely on the experienced psychological impact he or she is supposed to rate. The fact that the rater has, beyond the information in his psychological experience on $\mathrm{x}$, knowledge about from which stimulus the sensory experience he is asked to rate arose enables him to set his or her criterion $\mathrm{x}_{\mathrm{c}}$ at different places on $\mathrm{x}$, conditional upon whether $S_{1}$ or $S_{2}$ was given (see Figure 2). If $S_{1}$ was presented, he or she may set it at $x_{c} \mid S_{1}$, if $S_{2}$ was presented, at $x_{c} \mid S_{2}$. If now, from the proportion of combined events, "high"' $S_{2}$ (or "true"| $S_{2}$ ) and "high"' $S_{1}$ (or "true" $\mid S_{1}$ ), the parameter $\mathrm{d}^{\prime}$ is estimated, a bias will occur because the SDT data reduction implies the assumption of one single criterion point. Figure 3 shows the assumed psychophysical basis with the two criteria $x_{c} \mid S_{1}$ and $x_{c} \mid S_{2}$ now falling on a single point, $x_{c}^{*}$. The resulting $\mathrm{d}^{*}$ is biased by the amount $\left(\mathrm{x}_{\mathrm{c}}\left|\mathrm{S}_{1}-\mathrm{x}_{\mathrm{c}}\right| \mathrm{S}_{2}\right)$; that is,

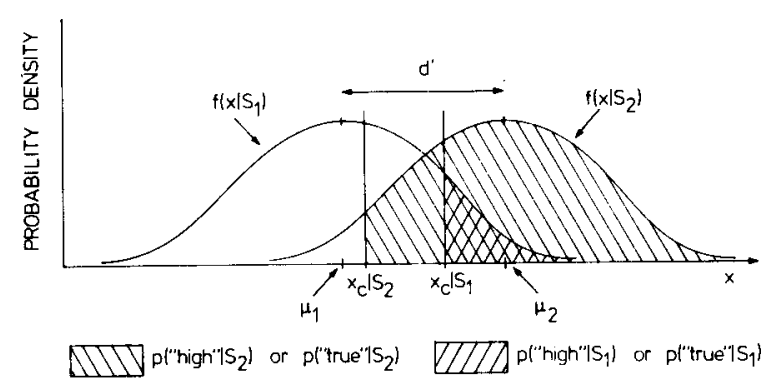

Figure 2. Psychophysical basis of ratings of psychological objects.

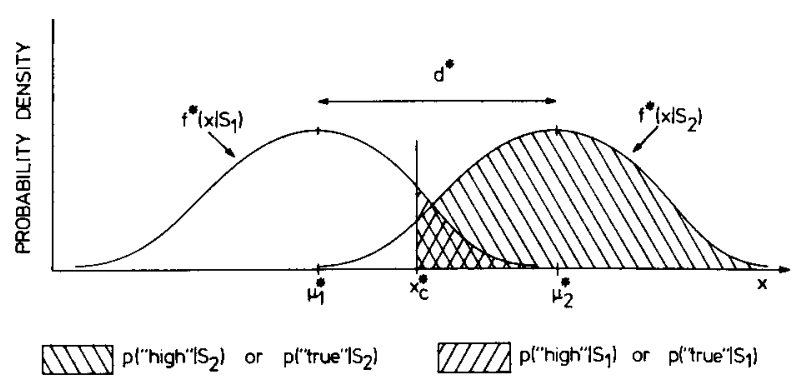

Figure 3. Apparent psychophysical basis of ratings of psychological objects resulting from the assumption of one single criterion as implied by SDT-type reduction of the data.

$$
d^{*}=d^{\prime}+\left(x_{c}\left|S_{1}-x_{c}\right| S_{2}\right) .
$$

It may be concluded that due to the psychophysical difference between the SDT discrimination and the common rating situation, signal detection type reduction of such rating data does not allow interpretation of resulting $\mathrm{d}^{\prime}$ values as unbiased psychological distances. To avoid misinterpretations, it might be worthwhile not to use the SDT notation for indices derived from psychological rating data.

\section{REFERENCE NOTE}

1. Chapman, C. R., \& Gentry, W. D. Assessment of emotion in heart attack patients: A signal detection theory approach to scaling. Unpublished manuscript, 1971. (Library, University of Washington, Seattle.)

\section{REFERENCES}

Chapman, C. R., \& Feather, B. W. Sensitivity to phobic imagery: A sensory decision theory analysis. Behavior Research and Therapy, 1971, 9, 161-168.

Clark, W. C., Kurlander, K., Bieber, R., \& Glassman, A. H. Signal detection theory treatment of response set in mood questionnaires. In I. G. Sarason \& C. D. Spielberger (Eds.), Stress and anxiety (Vol. 4). New York: Wiley, 1976.

Dorfman, D. D., \& Saslow, C. F. Application of signal detection theory to personality testing. In W. C. Clark (Chm.), Statistical decision theory: A new approach to problems in personality, social and clinical psychology. Proceedings of the American Psychological Association, 1972, 7, 904-905.

Green, D. M., \& Swets, J. A. Signal detection theory and psychophysics. New York: Wiley, 1966.

Grossberg, J. M., \& Grant, B. F. Clinical psychophysics: Application of ratio scaling and signal detection methods to research on pain, fear, drugs, and medical decision making. Psychological Bulletin, 1978, 85, 1154-1176.

Neufe LD, R. W. J. Effect of cognitive appraisal on $\mathrm{d}^{\prime}$ and response bias to experimental stress. Journal of Personality and Social Psychology, 1975, 31, 735-743.

(Received for publication August 15, 1978; revision accepted March 13, 1979.) 